\title{
Structure of the zodiacal cloud: new analytical and numerical solutions
}

\author{
N. Gor'kavyi ${ }^{1}$, L. Ozernoy ${ }^{2}$, J. Mather ${ }^{3}$, and T. Taidakova ${ }^{4}$ \\ ${ }^{1}$ Crimean Observatory, Ukraine, \\ ${ }^{2}$ George Mason U. and GSFC/NASA, U.S.A. \\ ${ }^{3}$ GSFC/NASA, U.S.A. \\ ${ }^{4}$ Crimean Observatory, Ukraine
}

(Received November 17, 1997; Revised April 13, 1998; Accepted April 13, 1998)

\begin{abstract}
Recent results of analytical and numerical modelling of the interplanetary dust (IPD) distribution are described. They have been obtained with a new techniques employing the continuity equation written in the space of orbital coordinates. A 3-D structure and the corresponding 2-D slices for the IPD cloud governed by the Poynting-Robertson drag are computed in the framework of our 'reference model', accounting for almost all of the known major sources of dust (5000 asteroids and 217 comets). We discuss also the origin and structure of the 'dust bands' and the resonant ring near Earth.
\end{abstract}

\section{Introduction}

Both dynamics and evolution of the interplanetary dust (IPD) flow can be conveniently described by the continuity equation written in the space of orbital coordinates, such as semimajor axis $a$, eccentricity $e$, inclination $i$, etc. (Gor'kavyi et al., 1996, 1997a). In a more recent work (Gor'kavyi et al., 1997b), we have applied this kinetic approach to explore the Poynting-Robertson drag as the leading effect for dynamics of small dust particles. Knowledge of the new integrals of motion enabled us to compute a 'reference model' for the 3-D structure of the zodiacal cloud produced by 217 comets (Marsden and Williams, 1996) and 5000 asteroids (ITA, 1994). Our reference model yields the dust density distribution up to a few AU in heliocentric distance $R$ and in geocentric altitude $Z$, which both turn out to be fairly consistent with the observational data. In particular, we have reproduced the classical solution $n(r) \propto r^{-1}$ that approximately represents the overall distribution of dust in the Solar system. We have also investigated various factors that could be responsible for the deviations of the power law index from -1 , including the influences of the orbital characteristics of dust sources, the evaporation of dust particles, as well as mixtures of dust particles of both asteroidal and cometary origin (Gor'kavyi et al., 1997b). Our approach enables us to compute detailed $(R, Z)$-sections and other characteristics of the zodiacal cloud. The reference model, supplemented by a model of drifting tori formed from recent collisional events, reveals 'band' features consistent with the observed dust bands at $1.5^{\circ}$ and $10^{\circ}$ (Gor'kavyi et al., 1997c).

More recently, we have improved our reference model by including those $\sim 10 \%$ of particles which are subjected to resonant effects. Using the improved reference model, we compute the distribution of resonant particles in the zodiacal cloud near Earth and then, using our transformation

Copy right (C) The Society of Geomagnetism and Earth, Planetary and Space Sciences (SGEPSS); The Seismological Society of Japan; The Volcanological Society of Japan; The Geodetic Society of Japan; The Japanese Society for Planetary Sciences. technique, make a transition from the space of orbital coordinates to the $(R, Z)$-space, which gives us the structure of the resonant ring near Earth. In the present paper, we report some results of our recent work.

\section{Theoretical Approach}

The underlying idea of our approach is rather simple. As distinct from the (cylindrical) coordinate $(R, Z)$-space, where the motion of IPD particles is usually very complicated (even if the PR-drag alone is taken into account), in the space of orbital elements, such as in the $(a, e)$-space, the motion of particles under all major effects occurs usually along either straight lines or some simple curves. Therefore the proposed approach includes the two major steps:

(I). Analysis of IPD particle dynamics in the $(a, e)$-space; and

(II). Transformation of the results into the $(R, Z)$-space.

For analysis of IPD particle dynamics, the most effective is the kinetic approach that makes use of the particle distribution function (d.f.). Solving the kinetic equation and using a hydrodynamic approach to particles orbiting a central massive body was done, in the coordinate space, by several investigators, including one of the authors of the present article (Gor'kavyi, 1986; Gor'kavyi and Fridman, 1994). Later on, as a systematic approach to the analysis of the zodiacal cloud, we proposed to employ the continuity equation in the space of orbital variables, in particular in the $(a, e)$-space (for detail, see Gor'kavyi et al., 1996, 1997a,b).

The second major step, after evaluating the d.f. in the $a, e, i$-space, is to compute the number density of particles in the $(R, Z)$-space. To this end, we have elaborated a novel transformation techniques.

After publishing our basic results (Gor'kavyi et al., 1996, 1997a,b), we have found that we omitted an earlier discussion of the kinetic equation for d.f. of interplanetary particles in the $(a, e, i)$-space, which was contained in the two comprehensive papers (Leinert et al., 1983; Banaszkiewicz et al., 1994). However, our approach has important advantages 


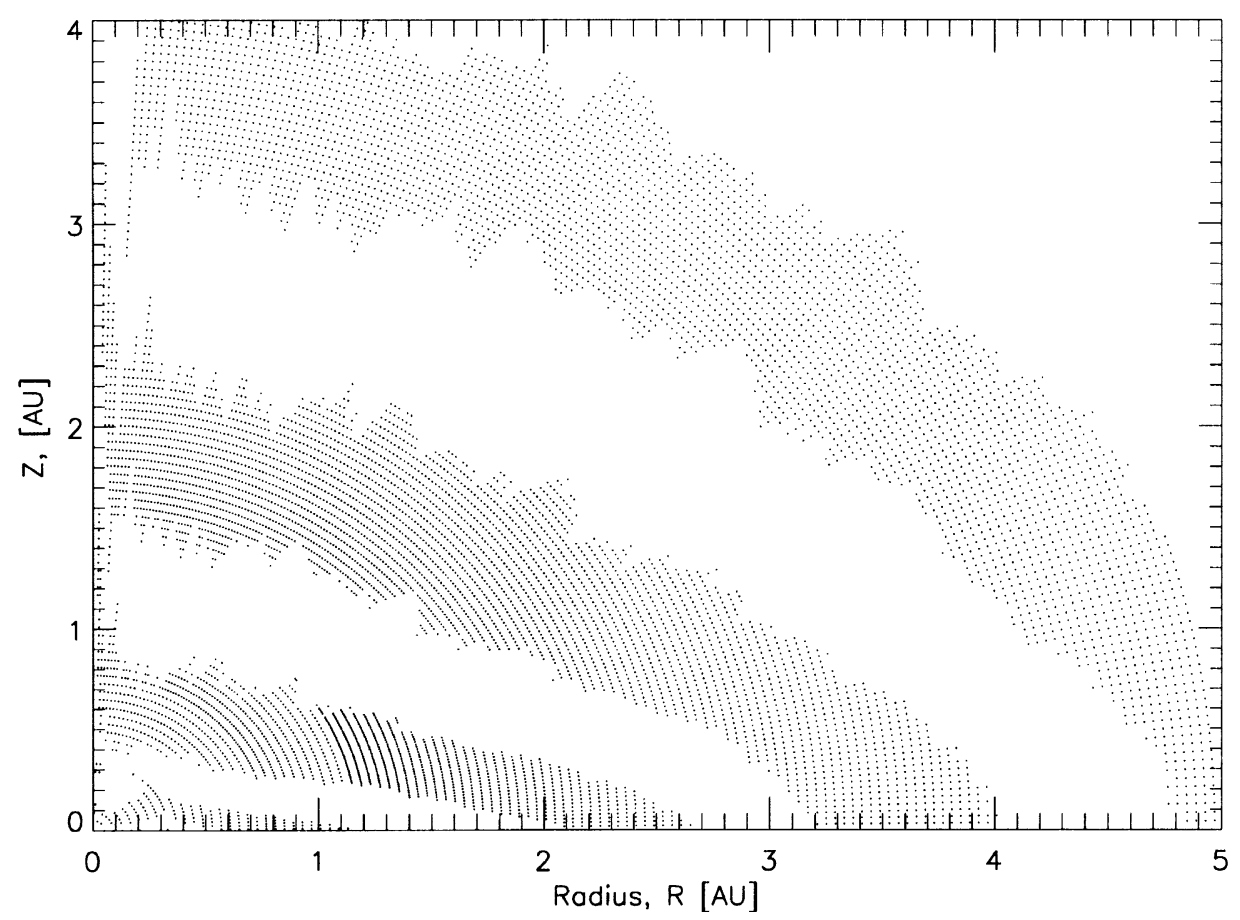

Fig. 1. A $(R, Z)$-density profile of the superposition of the two dust components formed by 5000 asteroids and 217 comets altogether, as it follows from our 'reference model'. Densities in the adjacent regions are differ $e$ times from each other.

compared to those papers:

- while doing step (I), we have obtained a simple analytical solution of our continuity equation both in the general case (where we have found two integrals of motion) and in the practically important case of point (in the $(a, e)$-space) dust sources;

- while doing step (II), we have found an analytical solution for the mass of dust in each cell of the $(R, Z)$-space. Our transformation techniques is much simpler than the complicated Haug's (1958) equations since it employs simple analytical expressions derived, for the first time, by Kessler (1981).

Using new analytical solutions in both steps (I) and (II) has enabled us to simplify radically the calculations, which made it possible to compute in a fast and effective way the structure of the zodiacal cloud, accounting for almost all known sources of dust (5000 asteroids and 217 comets). To give a particular example: the 'dust band' models are computed by our method a factor of 1000 faster compared to that in the framework of a purely numerical model, e.g., employed by the Florida group (Dermott et al., 1998).

\section{Results}

We have demonstrated the feasibility of the proposed method as well as a high efficiency of our techniques by computing a 3-D structure of the IPD cloud as it follows from our reference model. The corresponding 2-D slices of the dust distribution shown in Figs. 1 and 2 reveal both global and local features. Radial 'spokes' seen in Figs. 1 and 2 result from inhomogeneities of the source distribution, such as compact asteroidal groups or comets of a particularly high dust-production rate. A ring-like feature seen in Fig. 1 at $\simeq 0.30-0.35 \mathrm{AU}$ seems to be associated with an enhanced concentration of the cometary pericenters in that region as it reveals in Gor'kavyi et al. (1997b).

Our reference model is based on a few major assumptions: (i) all asteroids as the sources of dust are of an identical dust production rate; (ii) each comet produces dust particles whenever it passes its pericenter (the more intensively the smaller is the distance to the Sun); and (iii) all sources of dust are steady and, as a result, form steady 'plumes'. As an opposite limiting case, we have also considered a nonstationary dust production (e.g., by very recent asteroidal collisions). In this case, no 'plumes' are formed. Instead, the produced dust particles keep the initial orbital parameters $\{a, e, i\}$ and form slowly drifting tori. The distribution of dust in the cloud produced by superposition of all such asteroidal tori is shown in Fig. 3.

It is of interest to test whether the above models, both stationary and non-stationary ones, are capable of reproducing any of the observed 'dust bands' (DBs). The 10-fold magnified differences between the computed curves and a 'smooth' analytical approximation are shown in the bottom part of Fig. 4. One can see a few pronounced peaks associated with both stationary and non-stationary phenomena; those peaks might be called stationary dust bands (SDBs) and non-stationary dust bands (NDBs), respectively. As distinct from the SDBs, the NDBs, owing to their recent origin, have not experienced any appreciable P-R drift. Both SDBs and NDBs contribute to a peak at $1^{\circ}$; a peak at $10^{\circ}$ is associated with a NDB; and a broad feature at $17^{\circ}$ could be due to a SDB. Generally, our results confirm an earlier suggestion (Dermott, 1993) that the DBs are due to collisions in some asteroidal families.

In order to compute the structure of the zodiacal cloud near Earth, we can also employ our reference model that accounts 


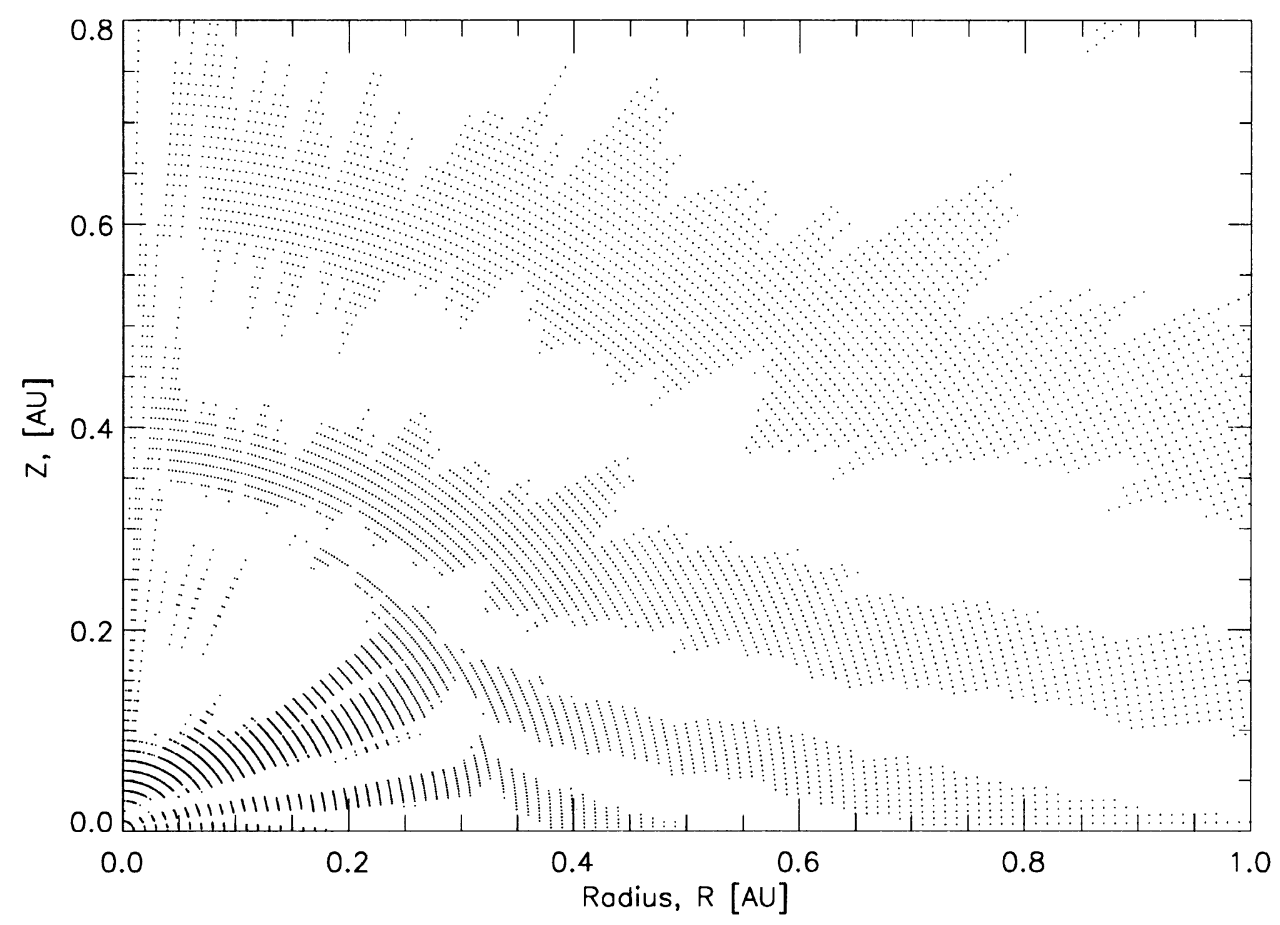

Fig. 2. An inner part of the IPD cloud computed according to our reference model. Densities in the adjacent regions are differ $\sqrt{e}$ times from each other.

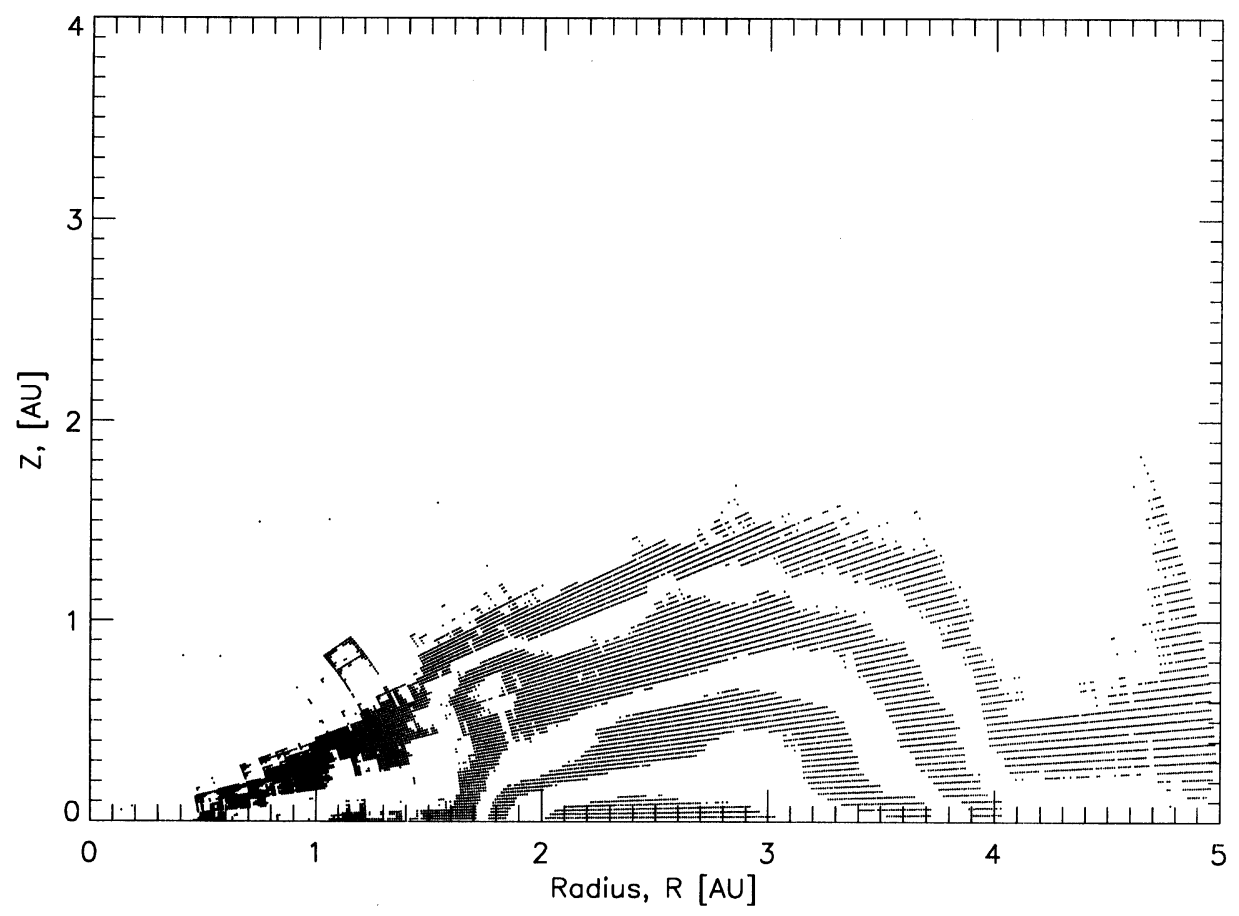

Fig. 3. A $(R, z)$-density profile of the superposition of dust tori formed by 5000 asteroids altogether. Densities in the adjacent regions are differ $e$ times from each other. An enhancement of dust density at $\sim 5 \mathrm{AU}$ is associated with the dust produced by the Trojans group of asteroids.

for the P-R drag, with the only difference that one needs to account for particle resonances near Earth. Although the corresponding near-Earth resonance dust ring predicted by Jackson and Zook (1989) has already been explored numerically in literature (e.g. Dermott et al., 1994), its structure was computed based on the evolution of a limited sample of par- ticles. Our 'reference model' enables us to find the particle's background distribution in the vicinity of each resonance and thereby to compute a detailed 3-D model with the account for the presence of particles captured into resonances.

In order to illustrate how our approach deals with resonant dust particles, we have performed, using some simpli- 


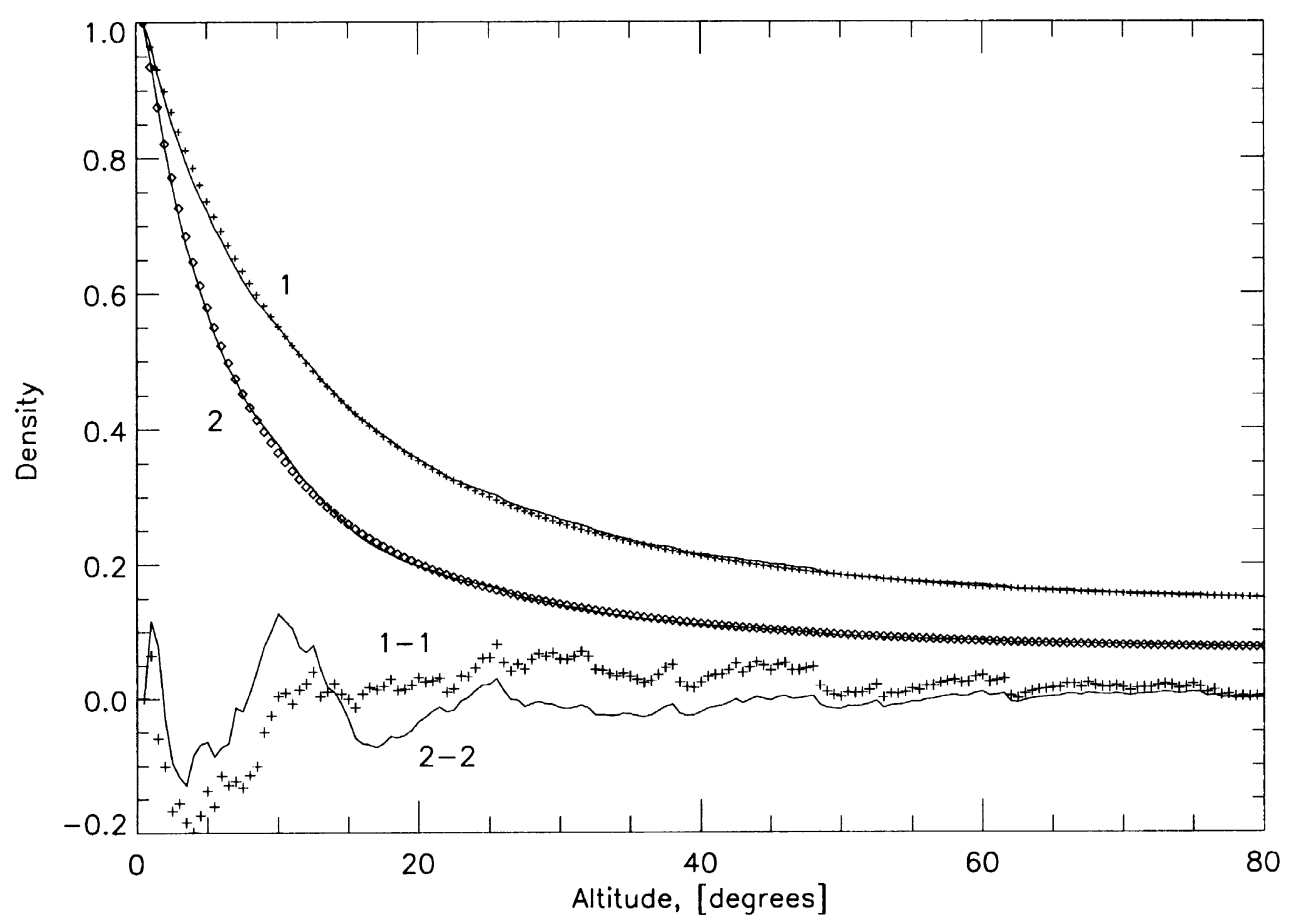

Fig. 4. Density of the zodiacal cloud in our reference model shown by solid curve 1 and in a mixture of the (stationary) reference model (50\%) and the (non-stationary) model of the drifting tori $(50 \%)$ shown by solid curve 2 as functions of ecliptic altitude in the elongation $90^{\circ}$. Each distribution is normalized to the density at $0^{\circ}$. Crosses and diamonds show an analytical approximation of the 'smooth' component. Large crosses (curve 1-1) and the lower solid curve 2-2 show a 10-fold magnified difference between the computed and 'smooth' components.

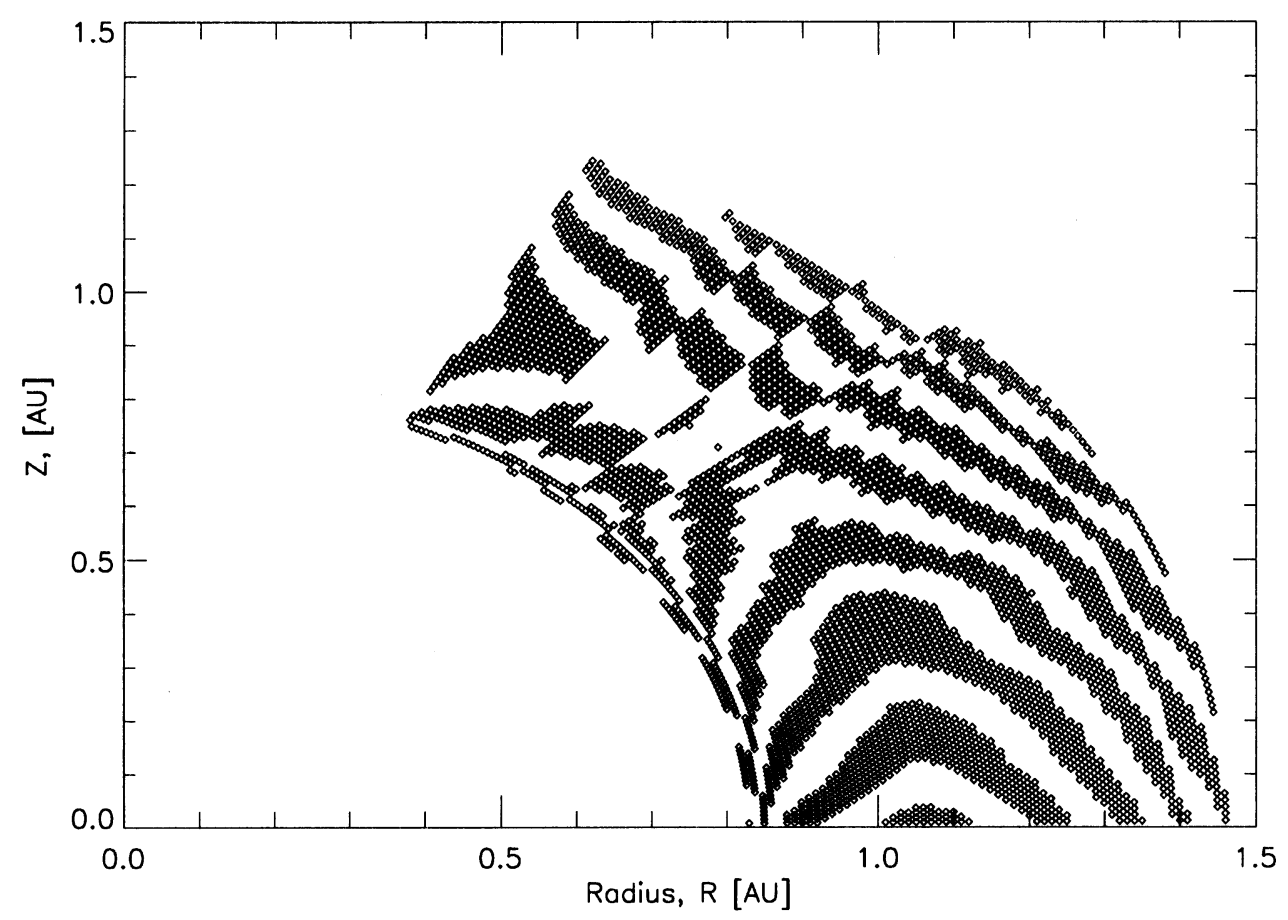

Fig. 5. A $(R, z)$-density profile of a computed resonance ring near Earth, which consists of dust particles produced by 5000 asteroids. Densities in the adjacent regions are differ $e$ times from each other. 


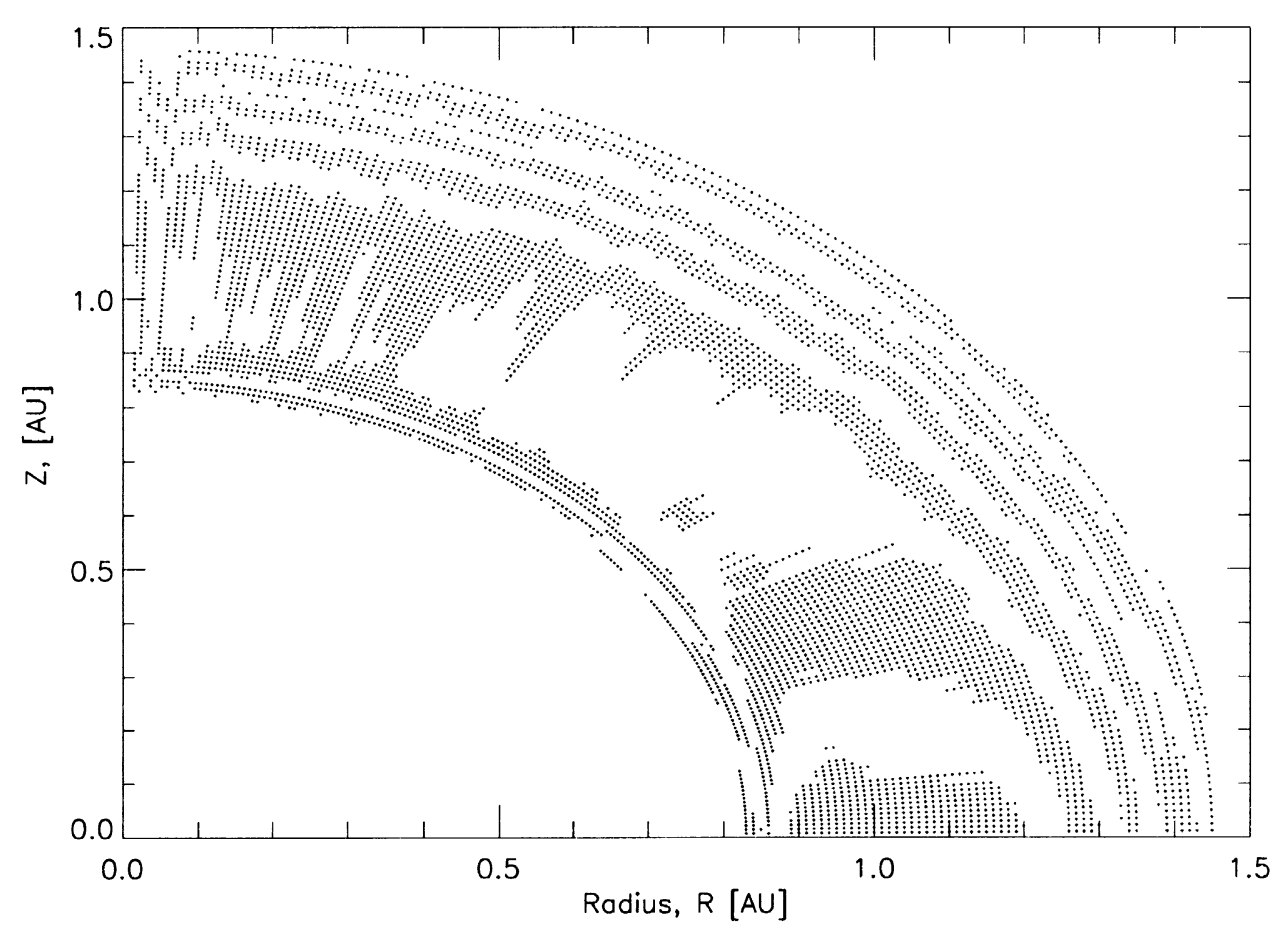

Fig. 6. $\mathrm{A}(R, z)$-density profile of a computed resonance ring near Earth, which consists of dust particles produced by 217 comets. Densities in the adjacent regions are differ $\sqrt{e}$ times from each other.

fying assumptions, trial calculations. We have employed analytical expressions for the resonant $e$-'heating' of particles (Weidenschilling and Jackson, 1993) and the numerical results on resonance trapping (Dermott et al., 1994) taken for the particles of $\beta=0.037, \beta$ being the ratio of the solar radiation pressure force to the gravitational force. The basic steps include:

(1) in the framework of our reference model, we have computed the distribution of non-resonant IPD particles in the regions of nine Earth resonances $p /(p+1)$ for $p=$ $4,5, \ldots, 13$;

(2) for the P-R 'plume' of each dust source, we have found a fraction of particles trapped, according to Dermott et al. (1994), to the corresponding resonant orbit, with accounting for the fact that the eccentricities of the trapped particles are within the range $e_{\min } \leq e \leq e_{\max }$;

(3) for each source of dust, we have solved 9 continuity equations for resonant particles moving in the $(a, e)$-space along the 9 lines $a_{p}=$ const;

(4) under a simplifying assumption that, for resonant particles, $i=$ const (actually, $i$ somewhat librates), we have found for each dust source $(a, e, i)$-distributions of resonant particles for 9 resonances $p /(p+1)$.

Those nine distributions, after summation over all dust sources, give a resonant ring whose $(R, Z)$-section at the Earth's location is shown in Fig. 6 for the dust of cometary origin and in Fig. 5 for the asteroidal dust. The overall shape and size of the resonant ring of asteroidal origin seem to be consistent with the DIRBE data (Dermott et al., 1994; Reach et al., 1995). However, we leave for a further work both a more detailed modelling and a confrontation with the data; while doing that modelling, one needs to employ the probabilities for the particle's capture into resonances to be calculated by direct numerical methods. An advantage of our approach is in ability to get a 3-D structure of resonant rings.

In the above 'reference model', we have assumed, for simplicity, all dust particles to be of the same size. It is worth noting that, although the particle's dynamics due to the P-R drag depends on the particle's size, our stationary 'reference model' for the structure of the IPD cloud does not contain any explicit dependence on it. While further improving our 'reference model' so as to make it multiparticle, one needs to account for the changes of the particles' initial orbital parameters since $\beta$ depends on a particle's size.

\section{Conclusions}

1. Our approach combining a new analytical method with numerical calculations enables us to compute a 3-D structure of the zodiacal cloud. The 2-D slices reveal interesting details (e.g. radial 'spokes', a ring-like structure at 0.3 AU, etc.) which need to be carefully examined in further detailed comparison with the data.

2. The (stationary) reference model, supplemented by a (non-stationary) model of drifting tori formed from recent collisional events, can be effectively used to examine the processes leading to the formation of 'dust bands'.

3. Our approach dealing with 1-D particle flows in the $(a, e, i)$-space can be successfully applied to explore the formation of the near-Earth resonant ring.

4. The particle number density of the cometary dust component in the resonant ring near Earth comprises less than 1\% compared to that of the asteroidal dust component. It might imply the dominating contribution of the asteroidal dust into the formation of resonant rings around the other planets.

5. In the simplest case when the probability of the par- 
ticle's capture into the outer resonance of Earth does not depend upon $i$, the maximum volume density of the resonant ring from the asteroidal dust in the ecliptic plane is as high as $50 \%$ of the background density of the asteroidal dust.

Our further plans include projecting of the entire structure of the zodiacal dust cloud onto the line of sight and a detailed comparison of the results with the COBE/DIRBE data.

Acknowledgments. We are grateful to Dr. A. Krivov and an anonymous referee for helpful comments. N.G. acknowledges the Zodiacal Science Workshop LOC for hospitality and financial support. T.T. has been supported by a Small Research Grant of the AAS from the Gaposchkin's Research Fund. L.O. is thankful to Janet Weiland for helpful discussions.

\section{References}

Banaszkiewicz, M., H. J. Fahr, and K. Scherer, Evolution of dust particle orbits under the influence of solar wind outflow asymmetries and the formation of the zodiacal dust cloud, Icarus, 107, 358-374, 1994.

Dermott, S. F. et al., Zodiacal dust bands, in Asteroids, Comets, Meteors, edited by A. Milani et al., p. 127-142, IAU: Netherlands, 1993.

Dermott, S. F., S. Jayaraman, Y. L. Xu, B. A. S. Gustafson, and J. C. Liou, A circumsolar ring of asteroidal dust in resonant lock with the Earth, Nature, 369, 719-723, 1994.

Dermott, S. F., K. Grogan, E. K. Holmes, and S. J. Kortenkamp, The dynamical structure of the zodiacal cloud, paper presented at ZCS workshop, Kobe, 1997.

Gor'kavyi, N. N., On the theory of linear oscillations of rotating collisional media, Sci. Inform. of Astron. Council of the USSR Acad. Sci., Riga, Zinatne, Iss. 61, 132-147, 1986 (in Russian).

Gor'kavyi, N. N. and A. M. Fridman, Physics of Planetary Rings. Celestial Mechanics of a Continuous Medium, 348pp., Nauka, Moscow, 1994; English translation: Springer-Verlag (in press).
Gor'kavyi, N. N., L. M. Ozernoy, and J. C. Mather, Dynamical evolution of interplanetary dust due to gravitational scattering on Jupiter and the inner planets, in Physics, Chemistry, and Dynamics of Interplanetary Dust, edited by B. Gustafson and M. Hanner, ASP Conf. Ser. 104, (San Francisco: ASP), p. 43, 1996.

Gor'kavyi, N., L. Ozernoy, and J. Mather, A new approach to dynamical evolution of interplanetary dust, Astrophys. J., 474, 496-502, 1997a.

Gor'kavyi, N., L. Ozernoy, J. Mather, and T. Taidakova, Quasi-stationary states of dust flows under Poynting-Robertson drag: New analytical and numerical solutions, Astrophys. J., 488, 268-276, 1997 b.

Gor'kavyi, N., L. Ozernoy, J. Mather, and T. Taidakova, Distribution of resonant particles in the zodiacal cloud: a new approach, Bull. AAS, 29, 782; Astrophys. J., 1997c (in preparation).

Haug, U., Überdie Häufigkeitsverteilung der Bahnelemente bei den interplanetaren Staubteilchen, Zeitschrift für Astrophysik, 44, 71-97, 1958.

ITA (Institute of Theoretical Astronomy), Ephemerides of Minor Planets, Nauka, St.-Peterburgh, 1994.

Jackson, A. A. and H. A. Zook, A solar system dust ring with the Earth as its shepherd, Nature, 337, 629-631, 1989.

Kessler, D. J., Derivation of the collision probability between orbiting objects: the lifetimes of Jupiter's outer moons, Icarus, 48, 39-48, 1981.

Leinert, L., S. Roser, and J. Buitrago, How to maintain the spatial distribution of interplanetary dust, Astron. Astrophys., 118, 345-357, 1983.

Marsden, B. G. and G. Williams, The Catalogue of Cometary Orbits, IAU Central Bureau for Astronomical Telegrams, Cambridge, Mass., 1996.

Reach, W. T. et al., Observational confirmation of a circumsolar dust ring by the COBE satellite, Nature, 374, 521-523, 1995.

Weidenschilling, S. J. and A. A. Jackson, Orbital resonances and PoyntingRobertson drag, Icarus, 104, 244-254, 1993.

N. Gor'kavyi (e-mail: gorkav@crao.crimea.ua), L. Ozernoy (e-mail: ozernoy@science.gmu.edu), J. Mather (e-mail: mather@stars.gsfc.nasa. gov), and T. Taidakova (e-mail: tat@crao.crimea.ua) 